\title{
Peginterferon-alpha 2a related peripheral neuropathy: A case report and review of the literature
}

\author{
Amalia Ghergu', Alexandra Oprisann ${ }^{1,2}$, Cristina Mitu', Sorana Popescu' ${ }^{1}$, Oana Morari ${ }^{1}$ \\ ${ }^{1}$ Clinic of Neurology, Colentina Clinical Hospital, Bucharest, Romania \\ ${ }^{2}$ Department of Neuroscience, "Carol Davila" University of Medicine and Pharmacy, \\ Bucharest, Romania
}

\begin{abstract}
We report the case of a 46-year old female with chronic hepatitis $C$ virus infection who developed paresthesia and weakness of face and upper and lower extremities nine months after administration of peginterferon-alpha 2a. Considering her clinical evolution, neurological examination and nerve conduction studies, she was diagnosed with multifocal motor neuropathy related to peginterferon-alpha treatment. The patient recovered after use of intravenous immunoglobulins. From our knowledge there is no data in the literature about multifocal motor neuropathy related to peginterferon-alpha treatment. Doctors should be aware of this rare association, which requires immediate drug discontinuation and early management.
\end{abstract}

Keywords: Peginterferon alpha, multifocal motor neuropathy, peripheral neuropathy, hepatitis C

\section{INTRODUCTION}

In 1957, Isaacs and Lindemann discovered that interferons (IFNs) had the ability to interfere with viral activity. IFNs are naturally occurring cytokines with immunomodulatory, antiproliferative, and antiviral properties (1). Presently, recombinant IFN- $\alpha$ forms are widely used in the treatment of chronic hepatitis $\mathrm{C}$ virus (HVC). IFN acts by binding to its receptor, thus activating secondary messengers, which results in multiple protein production important for the defense of the cell against viruses. Through its immunomodulatory effect it enhances antigen presentation to the immune system, which activates natural killer cells and other immune cells and increases production of cytokines. Antiviral effects are, among others, degradation of viral mRNA and inhibition of viral protein synthesis (1-3).

Peginterferon alfa-2a therapy is associated with a wide range of adverse effects, the most common being headache, insomnia, anxiety, flu-like symp- toms, leukopenia, thrombocytopenia, depression, and thyroid disorders (3). Few cases of peripheral neuropathy $(\mathrm{PN})$ have been reported $(<1 \%)$. Because these conditions are rare, there is currently no established consensus regarding their management. We report a case of peripheral neuropathy which developed after receiving interferon alpha treatment.

\section{CASE PRESENTATION}

A 46y old woman, diagnosed with hepatitis C infection in January 2016 for which treatment with peginterferon-alpha $2 \mathrm{a}$ and ribavirin was initiated in March 2016, is admitted to the Neurology Department in January 2017 for progressive weakness of the left leg and paresthesia followed a few weeks later by progressive weakness of the right leg. First symptoms were noticed in November 2016. The neurological examination revealed postural tremor in the upper limbs (mostly in the right one), right peripheral facial palsy, left ulnar nerve palsy, asym- 
metrical weakness of the lower limbs, predominantly distal (mostly in the left one), tendon reflexes were diminished, Hoffman and Babinski signs were absent, walking was impossible and no fasciculations were present. Sensation was normal for all modalities. Blood tests showed a mild normochromic normocytic anemia, mild increased of hepatic enzymes and LDH levels, negative HIV antibodies, absent cryoglobulines (CG), high CMV IgG levels $(4,934 \mathrm{U} / \mathrm{ml})$, positive CMV IgM and an undetectable viral load. Lumbar puncture was performed and CSF (cerebrospinal fluid) examination revealed normal glucose levels (44 mg/dl), high protein levels $(82 \mathrm{mg} / \mathrm{dl})$, positive Pandy's test and negative CMV IgG. WBC (white blood cell) count was 6.00 cells $/ \mathrm{mm}^{3}$. Nerve conduction studies showed evidence of multifocal motor neuropathy (MMN) conduction blocks. Conduction block, defined as loss of compound muscle action potential (CMAP) amplitude which exceeds $50 \%$ between 2 contiguous sites of stimulation (4), was identified in the left peroneal nerve, left and right median nerves and left ulnar nerve. Low amplitude CMAP was also seen in the right peroneal nerve, right tibial nerve and right ulnar nerve. The $\mathrm{F}$ waves were slightly prolonged in all the examined nerves. Sensory conduction studies were normal.

Considering the clinical, laboratory and electrophysiological findings, the patient was diagnosed with multifocal motor neuropathy. Peginterferon alpha-2a was immediately discontinued. The patient was treated with intravenous immunoglobulins $(0.4 \mathrm{~g} / \mathrm{kg}$ per day for 5 consecutive days) with an improvement of muscle strength within a few weeks.

\section{DISCUSSIONS}

The challenge in this case was the etiological diagnosis. Is the viral infection the cause of the neuropathy? Is this a complication of peginterferon-alpha treatment? Is there another possible explanation? About $40-75 \%$ of patients with hepatitis $\mathrm{C}$ have symptomatic peripheral neuropathy, especially when cryoglobulins are present. HCV is primarily correlated with type II cryoglobulinaemia. The most frequently associated polyneuropathies in hepatitis $\mathrm{C}$ infection are: sensory motor polyneuropathy that has been found in up to $9 \%$ of patients chronically infected with $\mathrm{HCV}$, prevalence that rises up to $30 \%$ in $\mathrm{HCV}$ with present cryoglobulines and also mononeuritis multiplex with a prevalence of $10 \%$. There are studies that characterize PN involvement in HCV with present cryoglobulines as a subacute, distal, motor sensory polyneuropathy. An asymmetrical sensory impairment has also been described $(7,8)$. In HCV infected patients, the presumed pathological mechanism is that nerve damage is secondary to epineurial vessels changes caused by occlusion or vasculitis induced by longstanding cryoglobulin precipitation with complement fixation and rheumatoid factor deposition that causes fascicular ischaemia which results in axonal degeneration. Also, it has been hypothesized that $\mathrm{HCV}$ may have a direct role in the pathogenesis of neuropathy: It could induce nerve damage by a direct cytopathic effect or by an immunomediated mechanism such as immune complex changes of the epineural vessels. This hypothesis seems to be supported by the finding of HCV-RNA in nerve biopsy specimens by RT-PCR in situ and could explain the neurological involvement in cryoglobulines negative patients. There is evidence of a vasculitic process both in $\mathrm{HCV} \mathrm{CG}+$ and $\mathrm{HCV}$ CGpatients. Regarding the pathophysiology of the vasculitic process in HCV CG- neuropathy, the activation of the complement may be attributable to three different mechanisms: the ability of the virus itself to activate the complement pathway, the reactivity of natural killer cells against the viral proteins and possibly the existence of an interaction between $\mathrm{HCV}$ and anti-HCV antibodies. Considering these mechanisms, a reduction in the viral load could lead to an improvement of symptoms $(7,8)$.

Interferon-alpha acts by binding with its specific receptor (IFNAR) found on the surface of the target cells, thus activating an intracellular signaling cascade, which takes the induction of IFN-stimulated genes (ISGs), which leads to the production of antiviral proteins such as 2' 5 '-oligoadenylate synthetase (2'5'OAS), protein kinase RNA, and Mx protein and establishes a non-virus-specific antiviral state inside the cell. The principal signaling mechanism used by IFN- $\alpha$ is the Janus kinase/signal transducers and activators of transcription (Jak/ STAT) pathway $(2,3)$. The mechanism through which interferon-alpha induces peripheral neuropathy is unknown, but there are studies suggesting an 
involvement of INF as a trigger factor of a pathological autoimmune reaction, such as molecular mimicry in immunologically-predisposed patients (9).

Assessing the likelihood of a causal connection between an environmental exposure - in our case, a drug - and an adverse event, the five primary elements of attribution reported by Miller et al. include:

1. temporal association;

2. lack of likely alternative explanations;

3. dechallenge;

4. rechallenge;

5. biological plausibility $(6,7)$.

The fourth element cannot be studied because peginterferon alpha $2 \mathrm{a}$ was not reinitiated. No alternative explanation was found. Nevertheless, temporal association and dechallenge (improvement in symptoms following discontinuation of the drug) suggests that peginterferon-alpha $2 \mathrm{a}$, rather than the hepatitis $\mathrm{C}$ infection, was related to the development of the neurological complication.

There have been described in the literature only a few cases of HCV infected patients with peripheral neuropathy related to interferon-alpha $2 \mathrm{a}$ treatment. Such examples of neuropathy are: acute inflammatory demyelinating polyneuropathy (AIDP), chronic inflammatory demyelinating neuropathy (CIDP), sensory neuropathy, Bell's palsy, autonomic neuropathy (10-19). One patient developed AIDP after a 16-week treatment with peginterferon alpha 2a. After discontinuation of interferon-alpha and after initiation of IVIG therapy, the patient's symptoms improved (10). Another patient developed AIDP after 5 weeks of peginterferon-alpha treatment. Discontinuation of interferon-alpha and symptomatic treatment lead to an improvement of symptoms after four weeks (11). Tellez-Zenteno et all describe a case in which a patient who received multiple cycles of interferon-alpha treatment developed paresthesias of both legs and was diagnosed with sensorymotor polyneuropathy (12). In the case report of Hirotani et all, a patient with chronic HCV infection developed CIDP after the initiation of IFN-alpha therapy. The treatment with interferon was withdrawn, but the neurological symptoms of this patient continued to progress; the patient's condition improved dramatically when IVIGs were given (13). In the case report of Lee MY et all, an HCV infected patient developed facial palsy after 3 weeks of interferon-alpha treatment. After discontinuation of interferon and after treatment with prednisolone, his palsy improved (14).

From our knowledge there is no data in the literature about MMN related to peginterferon-alpha treatment. MMN is a neurological demyelinating disorder which usually appears to be an isolated entity. It is a purely motor, asymmetric neuropathy with multifocal conduction blocks. The treatment of choice is IVIG, with a good response seen in as many as $80 \%$ of patients. The proposed mechanisms of action of IVIG is thought to be related to the inhibition or consumption of complement depositing found at nodes of Ranvier. Other agents such as cyclophosphamide, rituximab, mycophenolate mofetil, $\beta$-interferon, cyclosporine, azathioprine, and infliximab have been used for treatment of MMN (4).

\section{CONCLUSIONS}

Although rare, peginterferon-alpha 2a can cause peripheral neuropathies. Doctors prescribing peginterferon-alpha $2 \mathrm{a}$, as well as the neurologists, should be aware of such possible complications and ensure prompt withdrawal of the offending drug and appropriate therapy.

\section{REFERENCES}

1. Rijckborst V., Janssen H. The Role of Interferon in Hepatitis B Therapy, Curr Hepat Rep. 2010 Nov; 9(4): 231-238.

2. Robert M. Friedman M.D. Clinical uses of interferons. Br J Clin Pharmacol 2008 Feb; 65(2): 158-162

3. Sidney Pestka, The Interferons: 50 Years after their discovery, there is much more to learn. J. Biol. Chem. 2007, 282:20047-20051.

4. Victoria H. Lawson, David Arnold W. Multifocal motor neuropathy: a review of pathogenesis, diagnosis, and treatment, World $\mathrm{J}$ Gastroenterol. 2008 Jan 14; 14(2): 318-321.

5. Frederick W. Miller et al. Criteria for Environmentally Associated Autoimmune Diseases, J Autoimmun. 2012 Dec; 39(4): 253-258.

6. Rodriguez-escalera $\mathbf{C}$. et al. Multifocal motor neuropathy with conduction block in a patient with rheumatoid arthritis on infliximab therapy, Rheumatology 2005; 44:133-134

7. Santoro L. et al. Prevalence and characteristics of peripheral neuropathy in hepatitis $\mathrm{C}$ virus population, $J$ Neurol Neurosurg Psychiatry 2006; 77:626-629. 
8. Nemni R. et al. Peripheral neuropathy in hepatitis $C$ virus infection with and without cryoglobulinaemia, J Neurol Neurosurg Psychiatry 2003; 74:1267-1271

9. Levin M.C., Krichavsky M., Berk J. et al. Neuronal molecular mimicry in immune-mediated neurologic disease. Ann. Neurol. 1998; 44: 87-98.

10. Khiani V., Kelly T., Shibli A., Jensen D., Mohanty S.R. Acute inflammatory demyelinating polyneuropathy associated with pegylated interferon $\alpha 2 a$ therapy for chronic hepatitis $C$ virus infection. World J Gastroenterol 2008; 14(2): 318-321

11. Lahbabi et al. Acute inflammatory demyelinating polyneuropathy after treatment with pegylated interferon alfa-2a in a patient with chronic hepatitis $C$ virus infection: a case report, Journal of Medical Case Reports 2012, 6:278

12. Tellez-Zenteno J.F., Negrete-Pulido O., Nunez M., Estanol-Vidal B., Garcia-Ramos G. Peripheral neuropathy during a second cycle of treatment with high doses of interferon-alpha in a patient with hepatitis C, and a review of the literature. Rev Neurol 2002; 35: $644-646$

13. Hirotani M. et al. Chronic Inflammatory Demyelinating Polyneuropathy after Treatment with Interferon- $\alpha$, Inter Med 48: 373-375, 2009
14. Lee M.Y. et al. A Case of Bell's palsy associated with peginterferon alfa-2a and ribavirin therapy for chronic hepatitis $C$ virus infection, $J$ Hepatol 12 (3), 444-448. 92006

15. Lapinski T.W., Nowacka B., Michalewicz M. The peripheral polyneuropathy in patients with chronic HCV infection treated with peginterferon alfa with ribavirin. Pol Merkur Lekarski 2005; 18: $431-432$

16. Irioka T., Yamada M., Yamawaki M., Saito Y., Mizusawa H., Yamada M., Miura H. Acute autonomic and sensory neuropathy after interferon alpha-2b therapy for chronic hepatitis C. J Neurol Neurosurg Psychiatry 2001; 70: 408-410

17. Khiani V. et al. Acute Inflammatory Demyelinating Polyneuropathy Associated with Pegylated Interferon a 2a Therapy for Chronic Hepatitis C Virus Infection World J Gastroenterol 14 (2), 318-321. 2008

18. Melo C. et al. Basic aspects of the treatment for hepatitis $\mathrm{C}$ : mechanisms of action of interferon alpha and ribavirin and the bases of individualization, Intern Med. 2009; 48(5):373-5.

19. Hoare M., et al. Bell's palsy associated with IFN-alpha and ribavirin therapy for hepatitis $C$ virus infection.J Interferon Cytokine Res 2005; 25: $174-176$

Conflict of interest: none declared Financial support: none declared 